\title{
Control System of Underwater Vehicle Based on Neural Network
}

\author{
Chun-Meng JIANG ${ }^{1, \mathrm{a}}$, Lei WAN ${ }^{1, \mathrm{~b}}$, and Yu-Shan SUN ${ }^{1, \mathrm{c}}$ \\ ${ }^{1}$ State Key Laboratory of Autonomous Underwater Vehicle, Harbin \\ Engineering University, Harbin, 150001, China

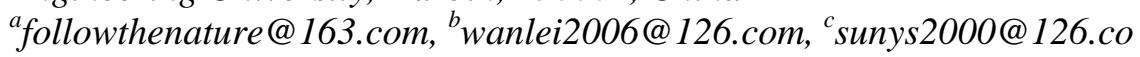 \\ $m$
}

\begin{abstract}
.
A Controller based on Neural Network (CNN) is introduced for motion control of UV. With this controller, an on-line studying method can be adopted without the sample data in several sample periods, resulting in high learning speed of the controller. The developed control system was applied to the 4-dimension control of a UV and succeeded in simulation platform. It is shown that the CNN has a great possibility to deal with the problems on the control system design of the future UV.

Keywords: Underwater Vehicle(UV), Neural Network, Control system.
\end{abstract}

\section{Introduction}

Owing to the unpredictable and high nonlinearity operating condition, many factors should be taken into account during the design of the control system of UV. Lots of control methods have been applied to UV such as fuzzy control, sliding mode control and so on[1-2].

Recently, neural network technologies have been applied to the field of UV. Yuh proposed a neural-network controller which used the desired velocity to train and adjust the controller. T. Fujii proposed a self-organizing neural-net-controller [3-5]. However, owing to the limitation of the sensors such as INS, the acquisition of the sample data and the training speed of the network become the bottleneck of this kind of controller.

A control system with neural network is introduced here. The aim is to show the feasibility of the control system. Section II describes the control scheme of the control system. In Section III, the application of the control system to the UV is described in detail. Section IV includes some experimental demonstrations.

\section{Controller Based on Neural Network (CNN)}

Figure 1 shows the proposed structure of CNN. The system includes three parts, real-time control part, parallel self-learning part and desired state programming part.

Four networks are included in the system, two control networks and two identification networks. To get the dynamic information, the two control networks 
have the structure shown in the left of Fig.2, while the two identification networks adopt the recurrent structure shown in the right of Fig.2.

From Figure 1, the data flow can be understood. The inputs to the real-time control network are the differences between the reference signals $r(t)$ and the state variables. The input to the real-time identification network is the output of the real-time control Network, $u(t)$, which is also the plant input. The desired output of the real-time identification network is the state variables. On the other hand, the inputs to the self-learning control network are the differences between the reference signals $r(k)$ and the state variables which are calculated by the self-learning identification network. The desired output of the control network is the result calculated by the desired state programming part.

Solving the identification and control networks, the processing of the ith neuron of the nth layer is shown as follows.

$$
\begin{aligned}
& v_{i}^{n}=\sum w_{i j}^{n-1} y_{j}^{n-1}+b_{i}^{n} \\
& y_{j}^{n}=\varphi\left(v_{i}^{n}\right)
\end{aligned}
$$

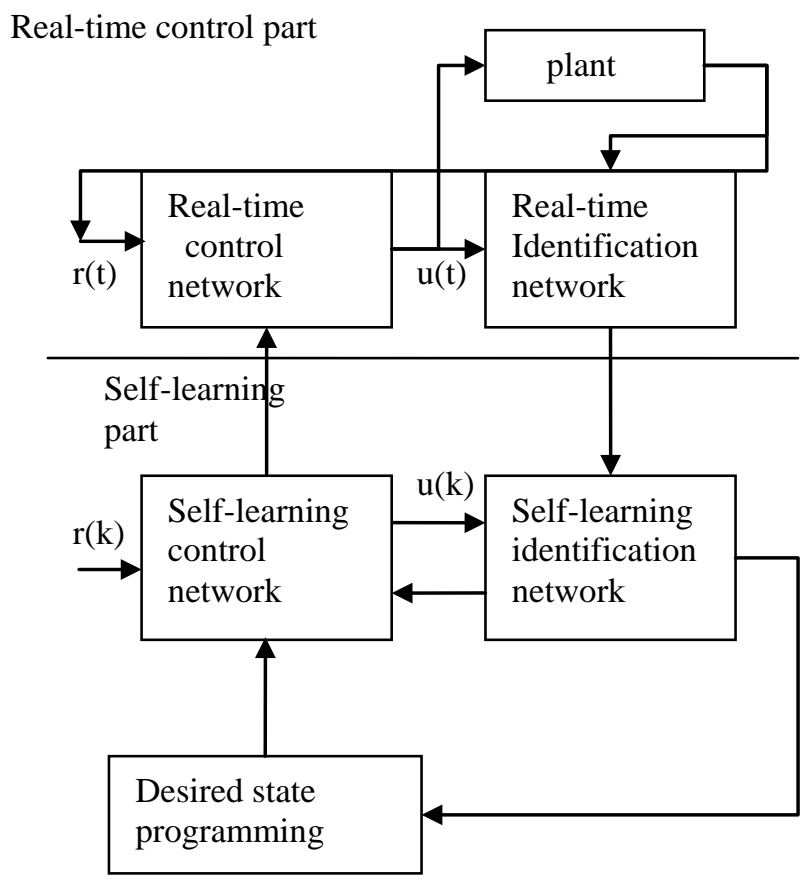

Fig.1 Structure of NNC 


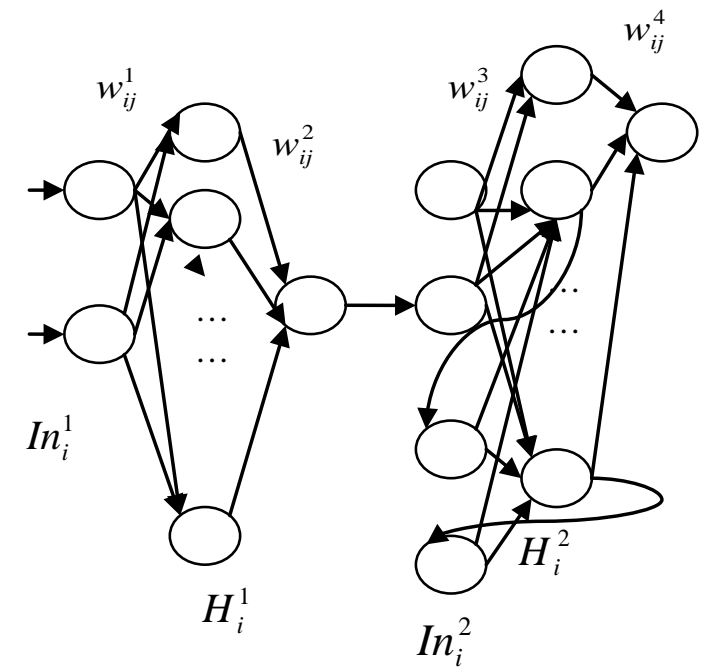

Fig.2 The network composed by an control network and an identification network

where $y_{j}^{n-1}$ is the output signal of neuron $\mathrm{i}$ in the previous layer n-1 and $w_{i j}^{n}$ is the synaptic weight of neuron $\mathrm{i}$ in layer $\mathrm{n}$ that is fed from neuron $\mathrm{j}$ in layer $\mathrm{n}-1 .{ }^{n}{ }^{n}$ is the bias adopted to the neuron i in layer n, and $\varphi(v)$ is a function which is given by Eq.2 and Eq.3:

input layer: $\varphi(v)=v$

others: $\quad \varphi(v)=(1-\exp (-v)) /(1+\exp (-v))$

The Identification networks and the Control networks are trained as a four-layer network shown in Fig.2.The Error Back-Propagation algorithm is adopted to train the network. A potential function $\mathrm{E}$ is given by Eq.4:

$$
E=\frac{1}{2} \sum\left(d_{i}-o_{i}\right)^{2}
$$


where $d_{i}$ is the ith element of the desired output, ${ }^{O_{i}}$ is the actual output. Each weight $w_{i j}^{n}$ should be changed by $\Delta w_{i j}^{n}$ to minimize E according to Eq.5:

$$
\Delta w_{i j}^{n}(p+1)=-\eta \frac{\partial E}{\partial w_{i j}^{n}}+\alpha \Delta w_{i j}^{n}(p)
$$

where $\Delta w_{i j}^{n}(p)$ is the value of weight changing in the pth step. $\alpha$ is the momentum constant, $\eta$ is the learning-rate parameter.

Let the ith neuron in the first hidden layer and the ith neuron in the second hidden layer be $H_{i}^{1}, H_{i}^{2}$ separately, and ith neuron in the first input layer and second input layer be $I n_{i}^{1}, I n_{i}^{2}$, and ith neuron in the first output layer be $u_{i}$

$$
\begin{aligned}
& \frac{\partial E}{\partial w_{i j}^{4}}=\left(\mathrm{d}_{k}-o_{k}\right) H_{j}^{2} \varphi^{\prime}\left(o_{i}\right) \\
& \frac{\partial E}{\partial w_{i j}^{1}}=\frac{\partial E}{\partial H_{j}^{1}} \frac{\partial H_{j}^{1}}{\partial w_{i j}^{1}}=\operatorname{In}_{j}^{1} \varphi^{\prime}\left(H_{i}^{1}\right) \sum_{n} w_{n j}^{2} \varphi^{\prime}\left(u_{n}\right) \frac{\partial E}{\partial u_{n}} \\
& \frac{\partial E}{\partial w_{i j}^{2}}=\frac{\partial E}{\partial u_{j}} \frac{\partial u_{j}}{\partial w_{i j}^{2}}=H_{i}^{1} \varphi^{\prime}\left(u_{i}\right) \sum_{m}\left[\varphi^{\prime}\left(H_{m}^{2}\right) w_{m j}^{3} \sum_{k}\left(\mathrm{~d}_{k}-o_{k}\right) \varphi^{\prime}\left(o_{k}\right) w_{k i}^{4}\right](8) \\
& \frac{\partial E}{\partial w_{i j}^{1}}=\frac{\partial E}{\partial H_{j}^{1}} \frac{\partial H_{j}^{1}}{\partial w_{i j}^{1}}=\operatorname{In}_{j}^{1} \varphi^{\prime}\left(H_{i}^{1}\right) \sum_{n} w_{n j}^{2} \varphi^{\prime}\left(u_{n}\right) \frac{\partial E}{\partial u_{n}}
\end{aligned}
$$

\section{Application to the Underwater Vehicle}

The performance of the CNN is investigated through the 4-degree of freedom (DOF) control on the simulation platform which is established basing on the hydrodynamic tank test on a UV. 


\section{Identification}

According to the maneuverability equation given as follows:
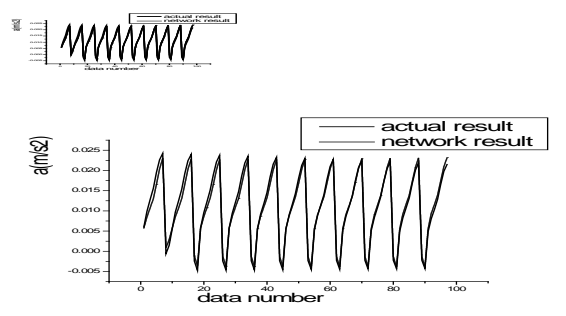

$\mathrm{m}$ is the mass of theUV and and gravity. The others are hydrodynamic coefficients acquired by tank experiment. is the thrust.

The identification network uses three layers. The input layer includes 10 neurons, two of which are the velocity and the thrust back of hidden layer. 8 neurons are adopted in hidden layer. Output is the acceleration The evaluation function is given by Eq.12:

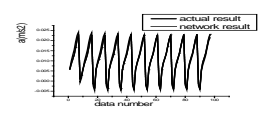

Four dimensions have been identified through the process discussed above. Fig.3 shows the identification results of the heave motion from the indicial response test. The training method presented above is performed off-line. The real-time identification network will be trained on-line, to get better identification result whose weight will be passed to the self-learning network to train the control network. 


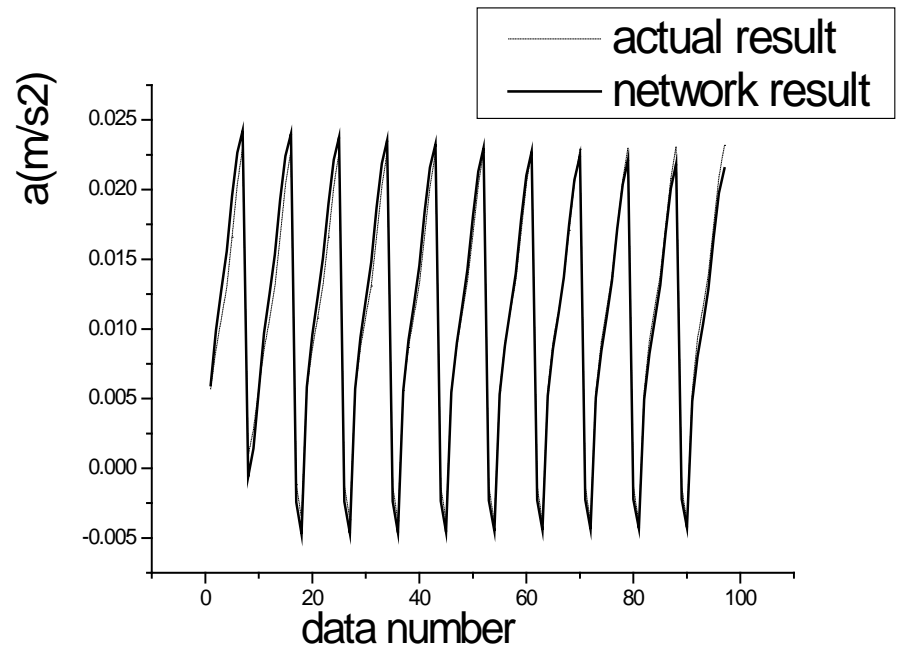

Fig.3 Identification result of heaving motion

\section{Control Network}

We use the desired acceleration as sample to train the control network online. The control network includes three layers, which includes two neurons, eight neurons, one neuron separately. By using the algorithm discussed in Section II, the self-learning control network is trained online. Here, the inputs are calculated by the self-leaning identification network.

\section{Experimental Demonstration}

To verify the validity of this CNN, plenty of computer simulation tests basing on the simulation platform have been finished. 4-DOF motion control tests are selected for demonstration as show in Figure 4. In the 4 DOF motion control test, the initial position is $\{0,0,1,0\}$, while the destination is $\{5,2,3,0.35\}$.

\section{Summary}

In this paper, a CNN system is developed, which can improve the training speed of the neural network controller. The feasibility of the CNN system is demonstrated by the application to the 4 DOF motion control of the simulation platform and shows good performance. It is shown that the proposed CNN system has ability to make highly reliable and robust control system for the UV and other nonlinear systems. 

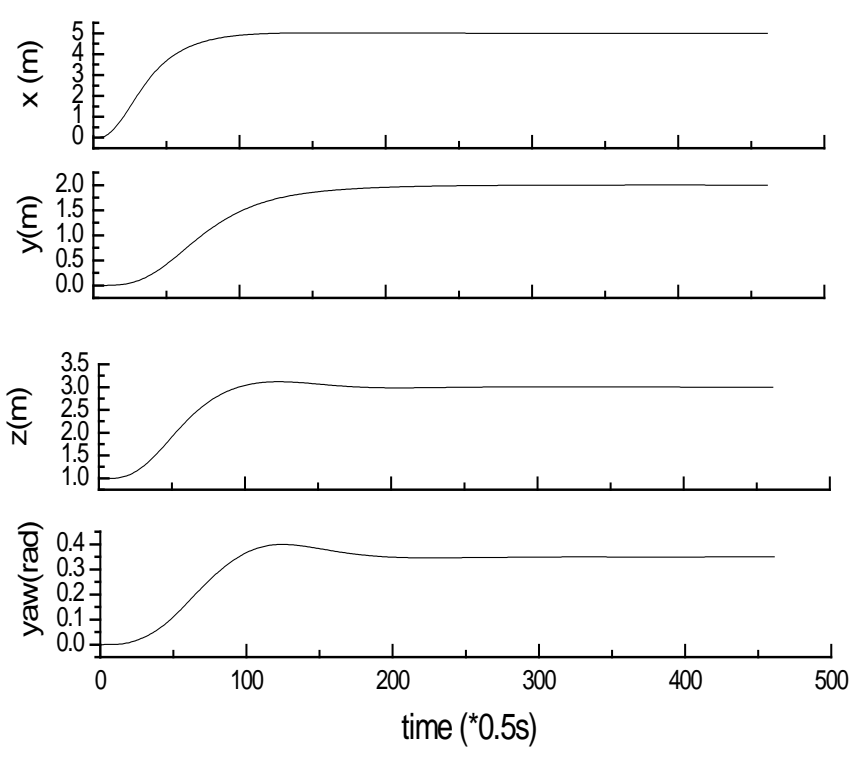

Figure 4. Simulation Result of the 4 DOF motion control

\section{References}

[1] Sun Yushan, Design of motion control of dam safety inspection underwater vehicle, J. Central South University, 19(2012)1522-1529.

[2] Wanlei, Research on Thruster Fault Diagnosis of Underwater Vehicles Based on NN, J. SHIPBUILDING OF CHINA, 4(2011)139-146.

[3] Wang Jianguo, An Improved S-Plane Controller for the Motion Control of Underwater Vehicles, J. Chinese Journal of Ship Research, 5 (2013)41-45.

[4] J. Yuh, A Neural Net Controller for Underwater Robotic Vehicles, IEEE J. Oceanic Engineering, 15 (2010)161-166.

[5] T.Fujii, Development of Motion Control system for AUV using Neural Nets, IEEE AUV, (2010) 81-86. 